\title{
TUBERCULOSIS OF SHORT TUBULAR BONES: A RARE PRESENTATION
}

Venkata Rama Chowdary ${ }^{1}$, P. Sunil Kumar², Rajendra Pal Singh B³,

\section{HOW TO CITE THIS ARTICLE:}

Venkata Rama Chowdary, P. Sunil Kumar, Rajendra Pal Singh B, "Tuberculosis of Short Tubular Bones: A Rare Presentation". Journal of Evolution of Medical and Dental Sciences 2014; Vol. 3, Issue 17, April 28;

Page: 4468-4472, DOI: $10.14260 /$ jemds/2014/2460

ABSTRACT: Tuberculosis of the metacarpals, metatarsals and phalanges is uncommon after the age of 5 years. It occurs in young children in endemic areas under 5 years of age. Tuberculosis of the short tubular bones like phalanges, metacarpals or metatarsals is quite uncommon beyond 6 years of age, once the epiphyseal centers are well established.

KEYWORDS: Tuberculosis, dactylitis, pseudotumour, granuloma, lymphadenitis.

INTRODUCTION: Tuberculous infection of metacarpals and metatarsals and phalanges of hands and feet is also known as tuberculous dactylitis. ${ }^{1,2}$

Hand is more frequently involved than the foot. During childhood short tubular bones have a lavish blood supply through a large nutrient artery entering in the middle of the bone. First inoculum of the infection is lodged in the centre of the marrow cavity and interior of the short tubular bone is converted virtually into a tuberculous granuloma. This leads to a spindle shaped expansion of the bone called as spinaventosa. ${ }^{3}$

Later sequestra will form with subperiosteal new bone formation. Abscess and sinus formationleads to secondary infection and further thickening of bone.

Radiologically affected bone appears as lytic lesion in the middle and subperiosteal new bone deposited. The cavity may contain soft coke like sequestra and takes a shape ofhoney-combing. Diffuse uniform infiltration or a cystic lesion.

CASE REPORT: A 9-year-old boy was brought by his mother, to KIMS hospital Amalapuram, with complaints of pain and swelling in the left index finger of six month's duration.

On examination, he had features of a draining abscess on the dorso-ulnar aspect of the proximal phalanx of the left index finger [Figure 1].

On detailed medical history, he admitted having evening raise of temperature and significant weight loss over the last three months. His general examination revealed emaciation with pallor. Rest of his general and systemic examination was unremarkable. The radiograph showed features of osteomyelitis in the proximal phalanx distal to the physis [Figure 2]. Chest X-ray was normal but tuberculin test was strongly positive about $14 \mathrm{~mm}$. Sedimentation rate was $65 \mathrm{~mm} /$ first hour. Aspirate from the small abscess around the sinus of the index finger showed no acid fast bacilli (AFB) on Ziehl Neelsen (ZN) staining and no pyogenic bacteria grew on culture. Abdominal ultrasonography was normal. A diagnosis of tuberculous osteomyelitis of the second proximal phalanx.

The patient was started on a four drug regimen (isoniazid + rifampicin + pyrazinamide + ethambutol for the first two months followed by isoniazid + rifampin for a total of seven months) as per DOT guidelines. Attention was also given to improve on his nutrition including high protein diet with vitamin supplements and hematinics. 


\section{CASE REPORT}

The involved finger is put functional position and rest is advised with active range of motion exercises was encouraged early. The patient was doing better with progressive healing of the bony lesion without any surgery. Patient was consistently gaining weight and hemoglobin level over months. In patients with unfavorable response or recurrence surgical debridement is required.

If a metacarpophalyngeal and metatarsophalyngeal interphalyngeal joints is ankylosed. Excision orthoplasty or corrective osteotomy is indicated.

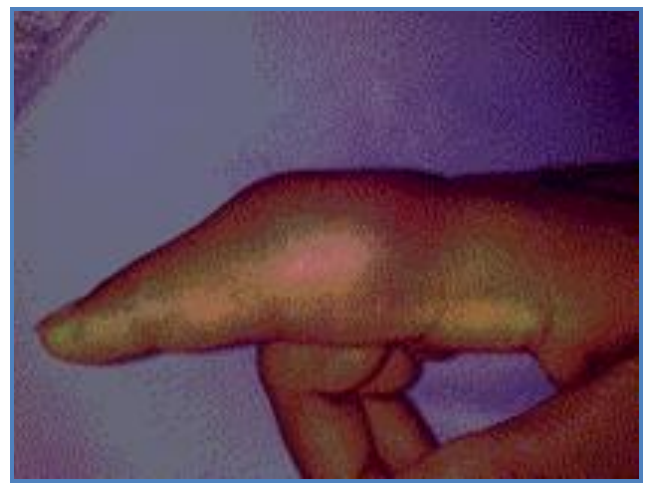

Figure 1: Fusiform swelling of tubercular dactylitis

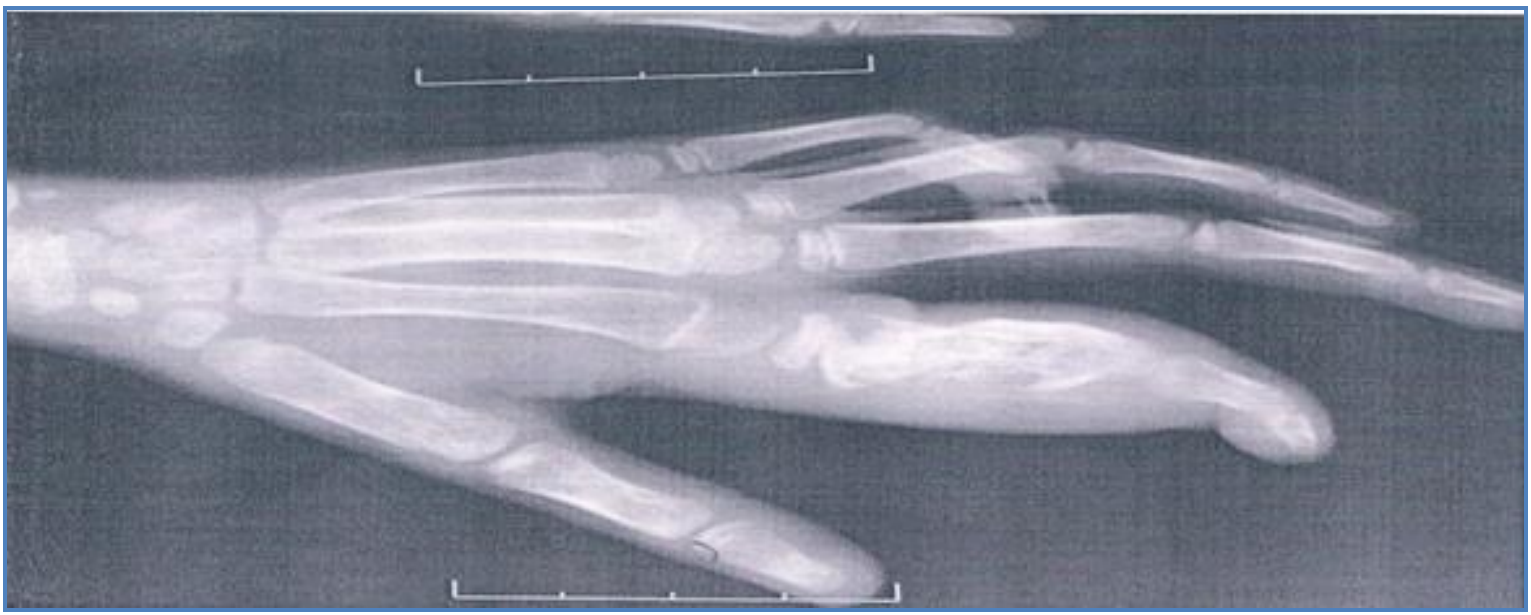

Figure 2: Index finger involving proximal and middlephalynges with thickening $\&$ sclerosis of bone.

DISCUSSION: Most tubercular infections of the bones are caused by the mycobacterium tuberculosis. Infection of the musculoskeletal system is a secondary disease caused by hematogenous spread from a primary lesion; it may occur shortly after the primary infection or years later as a disease reactivation. ${ }^{4,5}$

Tuberculous involvement of the metacarpals and phalanges is a rare presentation of extrapulmonary tuberculosis, especially in adults. ${ }^{4}$ A majority of $90 \%$ of patients with tubercular dactylitis are younger than 6 years of age. The short bones of the hand are the most frequent location 
of skeletal tuberculosis in infancy and early childhood before the epiphyseal centers are well established. At this tender age, the hematopoietic marrow in those bones offers a suitable environment for bacterial growth; pulmonary lesions usually can be demonstrated.

The infection rapidly involves the entire marrow space. Expansion of the bone with cystic quality is termed spinaventosa.

Radiological features: The affected bone appears expanded with lytic lesions in the middle (distal to the physis of proximal phalanx as seen in present case) and subperiosteal new bone formation along the involved bone.6,7 The cavity may contain soft coke like sequestra. Other findings on plain radiographs include osteopenia, soft-tissue swelling with minimal periosteal reaction, cysts in bone adjacent to joint and subchondral erosions. Typically, there is no periosteal layering or thickening, and sequestration ordinarily does not occur.8-10

In natural course, the disease may heal with shortening of the involved bone and deformity of the neighboring joint. Diaphyseal lesions tend to respond to therapy with slow healing. The bone density returns with slow filling of defects by new bone, which may become sclerotic, coarsely trabeculated, or relatively normal. The non-specific nature of these radiographic findings may potentially delay the diagnosis when spinaventosa is the lone clinical presentation.

Histopathology report, strongly positive tuberculin testA response to DOTs regime of ATT favored the diagnosis of tubercular scrofuloderma with tubercular dactylitis. There were only two cases of SCF with concomitant tuberculous synovitis out of which one was that of the thumb. The youngest female was 8-year-old and most cases occurred in the second decade (Mean age: 31 years). ${ }^{11}$ As mentioned earlier, spinaventosa is seen in children, mainly between the ages of 1 and 3 years. Our patient was also just 8-year-old; again showing the rarity of our case to have axillary SCF with concomitant spinaventosa.

The gold standard for the diagnosis of osseous tuberculosis remains the positive culture of mycobacterium tuberculosis from bone tissue.12 Differential diagnosis worth considering in such cases includes pyogenic osteomyelitis, Brodie's abscess, Atypical mycobacterial infection (due to mycobacterium scrofulaceum and M. avium-intracellulare), Actinomycosis or other deep mycosis (Sporotrichosis, Botryomycosis, Nocardiosis etc.), Kaposi's sarcoma and lueticdactylitis. ${ }^{13}$ There are recent published report of diagnostic dilemma and radical excision of such lesion as tumor in a young 2-year-old child.14 Presence of draining sinus in the tuberculous osteomyelitis may hinder its distinction from pyogenic disease. Clinically, pyogenic osteomyelitis tends to be acutely painful, swollen, and hot with fever.

Tuberculous osteomyelitis is relatively benign with mild pain and minimal pyrexia. The diffuse osteopenia associated with tuberculous infection may help distinguish it from pyogenic infection, as also the absence of sequestration. In syphilis, the bone is thickened by periosteal reaction. The definitive diagnosis relies on the detection M. tuberculosis by PCR or culture. ${ }^{14}$

Tuberculin test may provide useful supportive evidence in difficult cases. ${ }^{1}$ The optimal treatment duration for tubercular dactylitis remains unknown. Surgery has a limited role in the treatment in general but may play a supportive role, and curettage of the cavity has been recommended for avascular lesions. 15 


\section{REFERENCES:}

1. Pearlman HS, Warren RF. Tuberculous dactylitis. Am J Surg. 1961; 101:769-771. doi: 10.1016/0002-9610(61)90724-3.

2. Leung PC. Tuberculosis of the hand. The Hand.1978;10(3):285-291. doi: 10.1016/S0072968X(78)80052-7.

3. Andronikou S, Smith B. "Spinaventosa” tuberculous dactylitis. Arch Dis Child. 2002;86(3):206. doi: 10.1136/adc.86.3.206.

4. Subasi M, Bukte Y, Kapukaya A, Gurkan F. Tuberculosis of the metacarpals and phalanges of the hand. Ann Plast Surg. 2004;53(5):469-472. doi: 10.1097/01.sap.0000130708.80606.6a.

5. Coombs R, Fitzgerald RH. Infection in the orthopaedic patient. 1. Great Britain: Butterworth \& Co Ltd; 1989. pp. 297-298.

6. Agarwal S, Caplivski D, Bottone EJ. Disseminated tuberculosis presenting with finger swelling in a patient with tuberculous osteomyelitis: a case report. Ann Clin Microbiol Antimicrob. 2005;4:18. doi: 10.1186/1476-0711-4-18.

7. Al-Qattan MM, Bowen V, Manteo RT. Tuberculosis of the hand. J Hand Surg Br. 1994;19:234237. doi: 10.1016/0266-7681(94)90175-9.

8. Teo SY, Ong CL. Clinics in diagnostic imaging. Singapore Med J. 2006;47(3):243-249.

9. Vuyst D, Vanhoenacker F, Gielen J, Bernaerts A, Schepper AM. Imaging features of musculoskeletal tuberculosis. EurRadiol. 2003;13:1809-1819. doi: 10.1007/s00330-002-16096.

10. Yoon CJ, Chung HW, Hong SH, Kim CJ, Kang HS. MR findings of tuberculous dactylitis. Eur J Radiol. 2001;39(3):163-167. doi: 10.1016/S0720-048X(01)00327-8.

11. Wessels G, Hesseling PB, Beyers N. Skeletal tuberculosis: dactylitis and involvement of the skull. Pediatr Radiol. 1998;28:234-236. doi: 10.1007/s002470050339.

12. Padmavaty L, Lakshmana R, Ethirajan N, Krishnaswamy BK. Scrofuloderma: A clinicopathological and epidemiological study. Indian J Dermatol Venerol Leprol.2008;74:700.

13. Iftikhar U, Nadeem M, Aman S, Kazmi AH. Scrofuloderma: A common type of cutaneous tuberculosis. A case report. J Pak Assoc Dermatol. 2011;21:61-5.

14. Bandyopadhyay R, Mukherjee A, Mondal RK. Case Report: "Spinaventosa" tuberculous dactylitis in a 2 year old boy - A very rare disease. Open Orthop J.2012;6:118-20.

15. Ritz N, Connell TG, Tebruegge M, Johnstone BR, Curtis N. Tuberculous dactylitis: An easily missed diagnosis. Eur J Clin Microbiol Infect Dis.2011;30:1303-10. 


\section{CASE REPORT}

\section{AUTHORS:}

1. Venkata Rama Chowdary

2. P. Sunil Kumar

3. Rajendra Pal Singh B.

\section{PARTICULARS OF CONTRIBUTORS:}

1. Post Graduate, Department of Orthopaedics, Konaseema Institute of Medical Sciences \& Research Foundation, Amalapuram, East Godawary District, A. P.

2. Assistant Professor, Department of Orthopaedics, Konaseema Institute of Medical Sciences \& Research Foundation, Amalapuram, East Godawary District, A. P.

3. Professor, Department of Orthopaedics, Konaseema Institute of Medical Sciences \& Research Foundation, Amalapuram, East Godawary District, A. P.

\section{NAME ADDRESS EMAIL ID OF THE CORRESPONDING AUTHOR:}

Dr. P. V. Chowdary, S/o VenkataRao,

Pallakadiyam,

Rajanagaram (Mandal), East Godawary District.

E-mail: pathurisureshchowdary@gmail.com

Date of Submission: 05/04/2014. Date of Peer Review: 07/04/2014. Date of Acceptance: 08/04/2014. Date of Publishing: 23/04/2014. 